\title{
Integration and Simulations of INS/GNSS System Using the Approach of Carrier Phase Measurements
}

\author{
Khan Badshah, Qin Yongyuan
}

School of Automation, Northwestern Polytechnical University Xi'an, China

\begin{tabular}{l} 
Article Info \\
\hline Article history: \\
Received Jul 11, 2015 \\
Revised Oct 27, 2015 \\
Accepted Nov 12, 2015 \\
\hline Keyword: \\
Carrier Phase \\
Doppler \\
GNSS \\
INS \\
Kalman Filtering \\
MEMS \\
Pseudo Range \\
Simulation
\end{tabular}

\begin{abstract}
This paper discusses the techniques of attitude, velocity ad position estimation from GNSS carrier phase measurements, and investigates the performance of the lower precision MEMS based INS/GNSS system based on carrier phase measurements. Double differenced carrier phase measurements provide more accurate velocity and position estimation compared to code and Doppler measurements. However, integer ambiguity is required to be removed for precise positioning. Multiples antennae approach is used to derive the attitude information from carrier phase measurements in order to control the large initial misalignment angles for initialization of the integration process or to utilize during benign dynamics. Lever arm effect is considered to compensate for the separation of GNSS antenna and IMU location. The derived three GNSS observables are used to correct the INS through optimal Kalman filtering in a closed loop. Simulation results indicates the effectiveness of the integrated system for airborne as well as for land navigation vehicles.
\end{abstract}

Copyright (C) 2015 Institute of Advanced Engineering and Science. All rights reserved.

\section{Corresponding Author:}

Khan Badshah,

School of Automation,

Northwestern Polytechnical University Xi'an,

Shaanxi 710072, China.

Email: khan_phys@yahoo.com

\section{INTRODUCTION}

MEMS based inertial sensors have enabled the use of inertial technology in many applications that were not feasible in the past due to size and cost constraints. However, due to immaturity of this technology the performance of these sensors is limited which requires some non-traditional algorithms for improving their performance and reliability [1]. Nowadays a single-chip GNSS receiver are available in the market, which are being increasingly utilized in many navigation applications [2].The integration of GNSS and MEMS INS offers a very low cost, light weight and sufficiently accurate and reliable solution for navigation applications [3]. There are three main observables related to the GNSS called pseudo-range, carrier phase and Doppler measurements. These observables are used as a periodic updates to the INS for error estimation using the technique of optimal Kalman filtering. GNSS carrier phase measurements provide very accurate attitude, velocity and position data compared to the code phase and Doppler measurements. The attitude determination uses relative carrier-phase positioning between multiple antennas mounted on the same vehicle. GNSS attitude is very noisy, but does not drift, making it highly complementary to INS attitude and a solution to the heading calibration problem that occurs for some INS/GNSS applications [4]. By combining a lower precision MEMS INS with multiple antenna GNSS, a precise and stable attitude solution may be obtained. The inertial attitude solution can also aid the GNSS ambiguity resolution process by significantly reducing the search space. With a short baseline, the inertial attitude may completely resolve the ambiguity.

A full GNSS attitude solution requires three or more antennas. However, two antennas is sufficient for INS/GNSS where conventional INS/GNSS meets the roll and pitch accuracy requirements [5]. The 
accuracy of INS/GNSS attitude determination depends on the quality of the inertial sensors and the antenna separation. Longer lever arms produce more precise GNSS attitude measurements but can be subject to flexure [6,]. Generally, in the integration of INS/GNSS, the small angle approximation is applied to the attitude errors. This is usually valid for the roll and pitch attitude, which may be observed through levelling. However, the heading (or azimuth) angle is more difficult to find for initialization of the integrated system. Higher grades and expensive INS are only capable of gyro compassing. Similarly precise velocity and position can also be obtained from carrier phase measurements. Accurate velocity measurement plays an important role in many navigation applications such as automatic guidance and control of an unmanned aerial vehicle (UAV) and calibration of inertial navigation systems [7].

Conventionally a GNSS receiver estimates velocity by differencing two consecutive positions or by using Doppler measurements relative to receiver-satellite motion [8]. Doppler based velocity is the most widely used technique and usually has $\mathrm{m} / \mathrm{sec}$ accuracy, while the velocity obtained differencing consecutive positions from single point positioning has an accuracy one order of magnitude larger. When a greater accuracy is required, velocity can be estimated by processing differences of consecutive carrier phase measurements, which enables accuracies at the $\mathrm{mm} / \mathrm{s}$ level. The carrier phase ambiguity issue usually limits the use of this observable, but the time-differenced carrier phase technique overcomes this problem. Differencing two consecutive carrier phases greatly reduces the effects of various common mode errors between the measurements allowing very accurate velocity estimation. A limitation of carrier phase measurements is the reduced solution in harsh situations such as urban canyons because of the 4 satellites availability requirements [7].

The aim of this paper is the mathematical modelling for the estimation of attitude, velocity and position from GNSS carrier phase measurements and their performance analysis with INS through simulation results. The data of the two systems is fused through linearized Kalman filter using a loosely coupled approach. Simulation results for a lower grade MEMS IMU (STIM300) augmented with GNSS are produced to show the enhanced improvement in the navigating parameters.

\section{INS ERROR MODEL}

To transform the IMU and GNSS data to the user required navigation parameters, the relationship between different coordinate frames must be properly modelled. Each frame is considered as an orthogonal, right-handed co-ordinate frame. Definition of the frames used in this paper for the INS mechanization, error analysis and integration of INS/GPS are given below [4], [9-10].

a) Inertial frame (i-frame)

b) ECEF (e-frame)

c) Body frame (b-frame)

d) Navigation frame (n-frame)

Inertial frame ( $i$-frame) has its origin at the centre of the earth and is non-rotating with respect to the fixed stars, as shown in Figure 1. Earth Centred Earth Fixed (ECEF) frame ( $e$-frame) is a coordinate frame which has its origin at the earth's centre of mass and has axes which are fixed in the earth. Navigation frame ( $n$-frame) is a local level geodetic frame having origin at the mass centre of navigation system. East-NorthUp (ENU) frame is chosen as a local level navigation frame in this paper. The body frame ( $b$-frame) has origin coincident with the navigation frame and has three fixed axis defined as $\mathrm{x}$-(right), $\mathrm{y}$-(forward) and $\mathrm{z}$ (up). For angular motion, $\mathrm{x}$ is the pitch axis, $\mathrm{y}$ is the roll axis and $\mathrm{z}$-axis is the yaw axis. A linear error model of INS developed in $n$-frame is used to investigate the performance of the INS/GNSS integrated system using the carrier phase measurements of attitude, velocity and positions. Linearized error equations of attitude, velocity and position are given below [11].

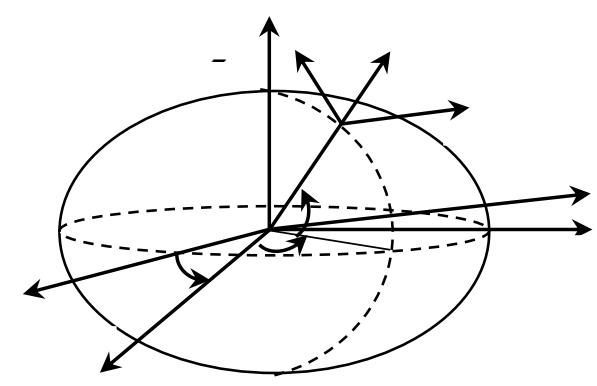

Figure 1. Inertial frame ( $i$-frame) 


$$
\left.\begin{array}{c}
\dot{\phi}_{E}=\left(\omega_{i e} \sin L+\frac{v_{E}}{R_{N h}} \tan L\right) \phi_{N}-\left(\omega_{i e} \cos L+\frac{v_{E}}{R_{N h}}\right) \phi_{U}-\frac{1}{R_{M h}} \delta v_{N}+\frac{v_{N}}{R_{M h}^{2}} \delta h-\varepsilon_{E} \\
\dot{\phi}_{N}=-\left(\omega_{i e} \sin L+\frac{v_{E}}{R_{N h}} \tan L\right) \phi_{E}-\frac{v_{N}}{R_{M h}} \phi_{U}-\omega_{i e} \sin L \delta L+\frac{\delta v_{E}}{R_{N h}}-\delta h \frac{v_{E}}{R_{N h N}^{2}}-\varepsilon_{N} \\
\dot{\phi}_{U}=\left(\omega_{i e} \cos L+\frac{v_{E}}{R_{N h}}\right) \phi_{E}+\frac{v_{N}}{R_{M h}} \phi_{N}+\left(\omega_{i e} \cos L+\frac{v_{E}}{R_{N h}} \sec ^{2} L\right) \delta L+\frac{\tan L}{R_{N h}} \delta v_{E}-\delta h \frac{v_{E} \tan L}{R_{N h N}^{2}}-\varepsilon_{U}
\end{array}\right\}
$$

$$
\left.\begin{array}{c}
\delta \dot{v}_{E}=\phi_{U} f_{N}-\phi_{N} f_{U}+\left[\frac{v_{N} \tan L-v_{U}}{R_{N h}}\right] \delta v_{E}+\left[2 \omega_{i e} \sin L+\frac{v_{E}}{R_{N h}} \tan L\right] \delta v_{N}-\left[2 \omega_{i e} \cos L+\frac{V_{E}}{R_{N h}}\right] \delta v_{U} \\
+2 \omega_{i e}\left[\left(v_{U} \sin L+v_{N} \cos L\right)+\frac{v_{E} v_{N}}{R_{N h}} \sec ^{2} L\right] \delta L+\left[\frac{v_{E} v_{U}-v_{E} v_{N} \tan L}{R_{N h}^{2}}\right] \delta h+\nabla_{E} \\
\delta \dot{v}_{N}=-\phi_{U} f_{E}+\phi_{E} f_{U}-\left[2 \omega_{i e} \sin L+\frac{v_{E}}{R_{N h}} \tan L\right] \delta v_{E}-\frac{v_{U}}{R_{M h}} \delta v_{N}-\frac{v_{N}}{R_{M h}} \delta v_{U}-\left[2 v_{E} \omega_{i e} \cos L+\frac{v_{E}^{2}}{R_{N h}} \sec ^{2} L\right] \delta L \\
+\left[\frac{v_{N} v_{U}}{R_{M h}^{2}}+\frac{v_{E}^{2} \tan L}{R_{N h}^{2}}\right] \delta h+\nabla_{N} \\
\left.\begin{array}{c}
\delta \dot{v}_{U}=\phi_{N} f_{E}-\phi_{E} f_{N}+\left[2 \omega_{i e} \cos L+\frac{2 v_{E}}{R_{N h}}\right] \delta v_{E}+\frac{2 v_{N}}{R_{M h}} \delta v_{N}-2 v_{E} \omega_{i e} \sin L \delta L-\left[\frac{v_{N}^{2}}{R_{M h}^{2}}+\frac{v_{E}^{2}}{R_{N h}^{2}}\right] \delta h+\nabla_{U} \\
\delta \dot{\lambda}=\frac{1}{R_{N h}} \sec L \delta v_{E}+\frac{v_{E}}{R_{N h}} \tan L \sec L \delta L-\frac{v_{E} \sec L}{R_{N h}^{2}} \delta h \\
\delta \dot{h}=\delta v_{U}
\end{array}\right\} \\
\delta \dot{L}=\frac{v_{N}}{R_{M h}^{2}} \delta h
\end{array}\right\}
$$$$
+2 \omega_{i e}\left[\left(v_{U} \sin L+v_{N} \cos L\right)+\frac{v_{E} v_{N}}{R_{N h}} \sec ^{2} L\right] \delta L+\left[\frac{v_{E} v_{U}-v_{E} v_{N} \tan L}{R_{N h}^{2}}\right] \delta h+\nabla_{E}
$$$$
\delta \dot{v}_{U}=\phi_{N} f_{E}-\phi_{E} f_{N}+\left[2 \omega_{i e} \cos L+\frac{2 v_{E}}{R_{N h}}\right] \delta v_{E}+\frac{2 v_{N}}{R_{M h}} \delta v_{N}-2 v_{E} \omega_{i e} \sin L \delta L-\left[\frac{v_{N}^{2}}{R_{M h}^{2}}+\frac{v_{E}^{2}}{R_{N h}^{2}}\right] \delta h+\nabla_{U}
$$

Where $\phi=\left[\begin{array}{lll}\phi_{E} & \phi_{N} & \phi_{U}\end{array}\right]^{T}$ is the attitude error; $\delta v^{n}=\left[\begin{array}{lll}\delta v_{E} & \delta v_{N} & \delta v_{U}\end{array}\right]^{T}$ and $\delta p=\left[\begin{array}{lll}\delta L & \delta \lambda & \delta h\end{array}\right]^{T}$ are velocity and position error vectors; $\varepsilon$ and $\nabla$ are gyro drift and accelerometer bias errors which are assumed to be constant

$$
\left.\begin{array}{l}
\dot{\varepsilon}^{b}=\left[\begin{array}{lll}
\dot{\varepsilon}_{x} & \dot{\varepsilon}_{y} & \dot{\varepsilon}_{z}
\end{array}\right]^{T}=0 \\
\dot{\nabla}^{b}=\left[\begin{array}{lll}
\dot{\nabla}_{x} & \dot{\nabla}_{y} & \dot{\nabla}_{z}
\end{array}\right]^{T}=0
\end{array}\right\}
$$

GNSS attitude, position and velocity parameters and their corresponding measurement models are derived in the following section. Accurate attitude determination of a body has been a challenge for precise navigation. Three non-collinear antennae are installed on the vehicle with known base line vectors in body frame, as shown in Fig-2. The relative carrier phase measurements produce the base line vectors between the antennas in ECEF frame [4]. The carrier phase measurements of the base lines vectors and the known base lines in body frames are processed to obtained the attitude matrix $C_{b}^{n}$ as

$$
r^{n}=C_{b}^{n} r^{b}
$$


Where $r^{b}$ and $r^{n}$ are constructed from vectors basis as

$$
\begin{aligned}
& r^{b}=\left[\begin{array}{lll}
r_{12}^{b} & r_{23}^{b} & r_{12}^{b} \times r_{23}^{b}
\end{array}\right]^{T} \\
& r^{n}=\left[\begin{array}{lll}
r_{12}^{n} & r_{23}^{n} & r_{12}^{n} \times r_{23}^{n}
\end{array}\right]^{T}
\end{aligned}
$$
frame as

Carrier-phase measurements give the vectors in ECEF frame which can be transformed into the n-

$$
r^{n}=C_{e}^{n} r^{e}
$$

Where $C_{e}^{n}$ transforms the vector from ECEF frame into navigation frame which can be calculated from precise GPS position as

$$
C_{e}^{n}=\left[\begin{array}{ccc}
\sin \lambda & \cos \lambda & 0 \\
-\sin L \cos \lambda & -\sin L \sin \lambda & \cos L \\
\cos L \cos \lambda & \cos L \sin \lambda & \sin L
\end{array}\right]
$$

The attitude matrix $C_{b}^{n}$ is then computed as

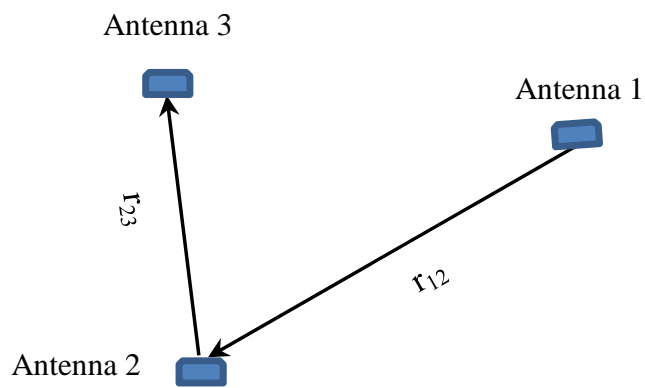

Figure 2. Antennae Scheme

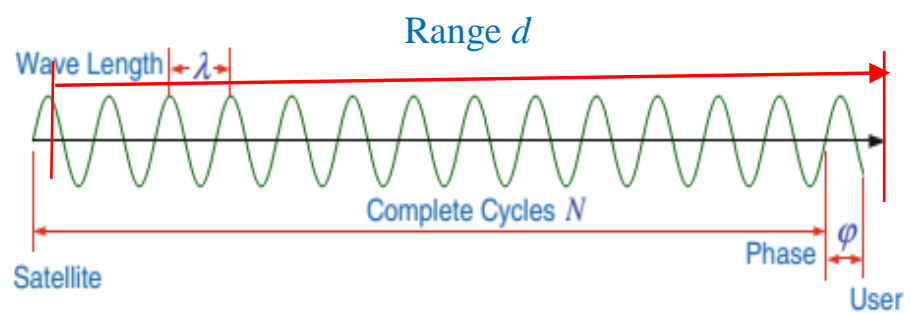

Figure 3. GPS Carrier Phase

$$
C_{b}^{n}=r^{n}\left(r^{b}\right)^{T}\left[r^{b}\left(r^{b}\right)^{T}\right]^{-1}
$$


Thus pitch, roll and yaw angles of the vehicle are

$$
\left.\begin{array}{l}
\theta=\sin ^{-1}\left[C_{b}^{n}(3,2)\right] \\
\gamma=\arctan 2\left[-C_{b}^{n}(3,1), C_{b}^{n}(3,3)\right] \\
\psi=\arctan 2\left[-C_{b}^{n}(1,2), C_{b}^{n}(2,2)\right]
\end{array}\right\}
$$

Generally, the derivate of receiver position is used to estimate the velocity of the vehicle which is very inaccurate method. Time-differenced carrier phase measurements at two successive epochs enables to estimate the velocity at the $\mathrm{mm} / \mathrm{sec}$ level accuracy [7]. The pseudo-range measurement $(\rho)$ can be written as

$$
\rho=d+c\left(\delta t_{u}-\delta t_{s}\right)+O_{\text {ion }}+E_{\text {eph }}+T_{\text {trop }}+\xi
$$

Where $d$ is the actual geometric receiver-satellite range as shown in Fig-3; $c$ is the velocity of light and $\delta t_{u}, \delta t_{s}$ are user and satellite clock errors; $O_{i o n}, E_{e p h}, T_{\text {trop }}$ are common mode errors as indicated; $\xi$ is the receiver white noise. The pseudo range and carrier phase measurements $(\varphi)$ are related as

$$
\rho=(N+\varphi) \times \eta
$$

Here $N$ is the total number of cycles as shown in Fig-3 which is called the integer ambiguity (IA); $\eta$ is the wave length of the carrier phase. Substituting Eq-12 in 11 and doing some rearrangement [12], we get

$$
\varphi=d+c\left(\delta t_{u}-\delta t_{s}\right)+\eta N-I_{\text {ion }}+E_{\text {eph }}+T_{\text {trop }}+\xi
$$

The difference between carrier phase measurements at two successive epochs $t_{j-1}$ and $t_{j}$ as shown in Fig-3, neglecting the small common mode errors and IA [7]

$$
\Delta \varphi_{\Delta t}=\Delta d+c\left(\Delta \delta t_{u}-\Delta \delta t_{s}\right)+\Delta \xi
$$

The satellite clock error is also corrected from ephemeris data

$$
\Delta \varphi_{\Delta t}=\Delta d+c \Delta \delta t_{s}+\Delta \xi
$$

All vectors shown in Fig-3 are expressed in ECEF frame. The difference between the receiversatellite range at two successive epochs is expressed as

$$
\begin{aligned}
\Delta d & =d\left(t_{j}\right)-d\left(t_{j-1}\right) \\
& =\left[r\left(t_{j}\right)-b\left(t_{j}\right)\right] . e\left(t_{j}\right)-\left[r\left(t_{j-1}\right)-b\left(t_{j-1}\right)\right] \cdot e\left(t_{j-1}\right)
\end{aligned}
$$

With

$$
e\left(t_{j}\right)=\frac{r\left(t_{j}\right)-b\left(t_{j}\right)}{\left\|r\left(t_{j}\right)-b\left(t_{j}\right)\right\|}, e\left(t_{j-1}\right)=\frac{r\left(t_{j-1}\right)-b\left(t_{j-1}\right)}{\left\|r\left(t_{j-1}\right)-b\left(t_{j-1}\right)\right\|}
$$

Where $\Delta d$ is related to the change in receiver position $\Delta b$ as [book].

$$
\Delta d=-e\left(t_{j}\right) \cdot \Delta b
$$

Substituting Eq-19 in 16 to obtain the adjusted carrier phase measurement 


$$
\Delta \varphi_{\Delta t}=-e\left(t_{j}\right) \cdot \Delta b+c \Delta \delta t_{u}+\Delta \xi
$$

There are four unknowns; $\Delta b=\left[\begin{array}{ccc}\Delta b_{x} & \Delta b_{y} & \Delta b_{z}\end{array}\right]^{T}$ and $c \Delta \delta t_{u}$ in 24, therefore 4 satellites, at least are required to be visible at two consecutive epochs for accurate velocity estimation. This is the main drawback of the carrier phase measurements. If there are $m$ number of visible satellites $(m \geq 4)$ then the four unknown parameters can be estimated using least square method.

$$
x=\left(H^{T} H\right)^{-1} H^{T} \tilde{z}
$$

Where

$$
\begin{aligned}
x= & {\left[\begin{array}{llll}
\Delta b_{x} & \Delta b_{y} & \Delta b_{z} & c \delta t_{r}
\end{array}\right]^{T}, z=\left[\begin{array}{llll}
\Delta \varphi_{1} & \Delta \varphi_{2} & \cdots & \Delta \varphi_{m}
\end{array}\right]^{T} } \\
H= & {\left[\begin{array}{cccc}
e_{1}^{x}\left(t_{j}\right) & e_{1}^{y}\left(t_{j}\right) & e_{1}^{z}\left(t_{j}\right) & 1 \\
e_{2}^{x}\left(t_{j}\right) & e_{2}^{y}\left(t_{j}\right) & e_{2}^{z}\left(t_{j}\right) & 1 \\
\vdots & \vdots & \vdots & \vdots \\
e_{m}^{x}\left(t_{j}\right) & e_{m}^{y}\left(t_{j}\right) & e_{m}^{z}\left(t_{j}\right) & 1
\end{array}\right] }
\end{aligned}
$$

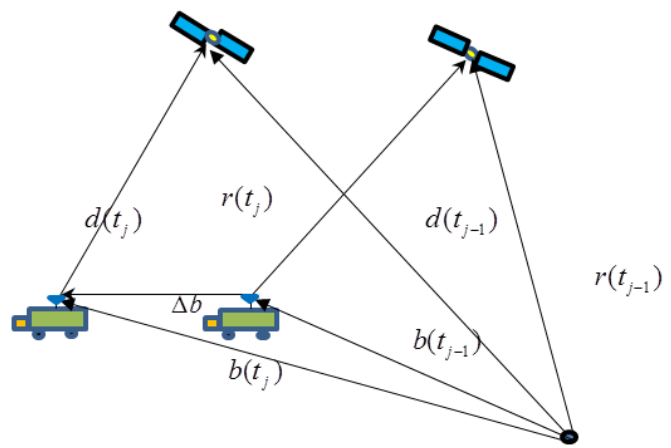

Figure 4. Satellite-receiver

The average velocity of the vehicle is obtained as

$$
\left\langle v_{e}\right\rangle=\frac{\Delta b}{\Delta t}
$$

This is the velocity in ECEF frame which can be transformed into the n-frame as

$$
v^{n}=C_{e}^{n}\left\langle v_{e}\right\rangle
$$

Positioning from carrier phase measurements is more precise than from code phase. Accuracy of centimetre level in position is attainable using the procedure of carrier phase measurements. However, the integer ambiguity is a critical issue to be resolved for precise positioning [4]. Many techniques have been researched and proposed to remove this ambiguity. As given in Eq-12, the carrier phase measurement is modelled as

$$
\tilde{\varphi}=d+c\left(\delta t_{r}-\delta t_{s}\right)-I_{i o n}+E_{e p h}+T_{\text {trop }}+\eta N+\xi
$$


Where $d$ is the true geometric range between the receiver and satellite as shown in Fig-3. The variables caped with " $\sim$ " and "^" represent respectively the measured and estimated quantities.

$$
d=\sqrt{\left(x_{s}-x_{r}\right)^{2}+\left(y_{s}-y_{r}\right)^{2}+\left(z_{s}-z_{r}\right)^{2}}
$$

Where $\left[\begin{array}{lll}x_{r} & y_{r} & z_{r}\end{array}\right]^{T}$ is the receiver's true position and $\left[\begin{array}{lll}x_{s} & y_{s} & z_{s}\end{array}\right]^{T}$ is the satellite position in ECEF frame. Equation-26 is a nonlinear function which can be linearized around a best estimated position of the receiver. Perturbing the receiver's position about the estimated position $\left[\begin{array}{lll}\hat{x}_{r} & \hat{y}_{r} & \hat{z}_{r}\end{array}\right]^{T}$

$$
x_{r}=\hat{x}_{r}+\delta x_{r}, y_{r}=\hat{y}_{r}+\delta y_{r}, z_{r}=\hat{z}_{r}+\delta z_{r}
$$

Substituting Eq-28 in 27 and simplifying

$$
d=\hat{d}+\frac{\left(x_{r}-x_{s}\right) \delta x_{r}+\left(y_{r}-y_{s}\right) \delta y_{r}+\left(z_{r}-z_{s}\right) \delta z_{r}}{\hat{d}}
$$

Here $\hat{d}$ is the estimated range. Using the value of $d$ in 26 and neglecting the common mode errors

$$
\tilde{\varphi}-\hat{d}=\frac{\left(x_{r}-x_{s}\right) \delta x_{r}+\left(y_{r}-y_{s}\right) \delta y_{r}+\left(z_{r}-z_{s}\right) \delta z_{r}}{\hat{d}}+c \delta t_{r}+\eta N+\xi
$$

Equation-30 is further simplified as

$$
\tilde{z}=A x+\xi
$$

Where

$$
\begin{gathered}
\tilde{z}=[\tilde{\varphi}-\hat{d}], x=\left[\begin{array}{lllll}
\delta x_{r} & \delta y_{r} & \delta z_{r} & N & c \delta t_{r}
\end{array}\right]^{T} \\
A=\left[\begin{array}{lllll}
\frac{\hat{x}_{r}-x_{s}}{\sqrt{\left(\hat{x}_{r}-x_{s}\right)^{2}}} & \frac{\hat{y}_{r}-y_{s}}{\sqrt{\left(\hat{y}_{r}-y_{s}\right)^{2}}} & \frac{\hat{z}_{r}-z_{s}}{\sqrt{\left(\hat{z}_{r}-z_{s}\right)^{2}}} & \eta & 1
\end{array}\right] \\
=\left[\begin{array}{lllll}
A_{x} & A_{y} & A_{z} & \eta & 1
\end{array}\right]
\end{gathered}
$$

It must be noted here that number of unknowns has increased by integer ambiguity. For four visible satellites $(m=4)$ at epoch $t$, equations 32 and 33 will modify as [12].

$$
\begin{aligned}
& x=\left[\begin{array}{llllllll}
\delta x_{r} & \delta y_{r} & \delta z_{r} & N^{1} & N^{2} & N^{3} & N^{4} & c \delta t_{r}
\end{array}\right]^{T} \\
& \tilde{z}=\left[\begin{array}{c}
\tilde{\varphi}^{1}-\hat{d}^{1} \\
\tilde{\varphi}^{2}-\hat{d}^{2} \\
\tilde{\varphi}^{3}-\hat{d}^{3} \\
\tilde{\varphi}^{4}-\hat{d}^{4}
\end{array}\right], A=\left[\begin{array}{rrrrrrrr}
A_{x}^{1} & A_{y}^{1} & A_{z}^{1} & \eta & 0 & 0 & 0 & 1 \\
A_{x}^{2} & A_{y}^{2} & A_{z}^{2} & 0 & \eta & 0 & 0 & 1 \\
A_{x}^{3} & A_{y}^{3} & A_{z}^{3} & 0 & 0 & \eta & 0 & 1 \\
A_{x}^{4} & A_{y}^{4} & A_{z}^{4} & 0 & 0 & 0 & \eta & 1
\end{array}\right]
\end{aligned}
$$




\section{SINS/GNSS INTEGRATION ALGORITHM}

In this integration algorithm the GPS derived attitude, position and velocity parameters are used as a measurement to the centralized Kalman Filter. The Kalman filter (KF) is the optimal estimator that minimizes the mean square error when the state and measurement dynamics are linear in nature, provided the process and measurement noises are modelled as white Gaussian. The SINS errors dynamic is a continuous time process governed by a linear stochastic equations.

$$
\begin{aligned}
& \dot{x}=F x+w \\
& z_{k}=H_{k} x_{k}+v_{k}
\end{aligned}
$$

Where $x$ is the state vector and $F$ is the dynamic matrix; $w$ and $v_{k}$ are process and measurement white noises. $F$ matrix is obtained by combining and rearranging the terms in Eqs-1, 2, 3 and 4 . The state vector is designed as.

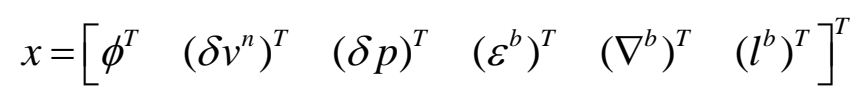

Where $l^{b}$ is the lever arm vector from INS to the GNSS antenna centre, expressed in body frame. The GNSS derived attitude, velocity and position are subtracted from INS data and used as a measurements to the ceneterlized LKF. The design matrix for attitude measurements is constructed here for fusing the data of the two systems. Define the attitude measurements as

$$
\begin{aligned}
& A_{g p s}=\left[\begin{array}{lll}
\theta_{g p s} & \gamma_{g p s} & \psi_{g p s}
\end{array}\right]^{T}, A_{i n s}=\left[\begin{array}{lll}
\theta_{i n s} & \gamma_{i n s} & \psi_{i n s}
\end{array}\right]^{T} \\
& \delta A=A_{i n s}-A_{g p s} \\
& {\left[\begin{array}{lll}
\delta \theta & \delta \gamma & \delta \psi
\end{array}\right]^{T}=\left[\begin{array}{lll}
\theta_{i n s} & \gamma_{i n s} & \psi_{i n s}
\end{array}\right]^{T}-\left[\begin{array}{lll}
\theta_{g p s} & \gamma_{g p s} & \psi_{g p s}
\end{array}\right]^{T}}
\end{aligned}
$$

The attitude difference is related to the attitude error vector as

$$
\left[\begin{array}{c}
\phi_{E} \\
\phi_{N} \\
\phi_{U}
\end{array}\right]=-C_{b}^{n} C_{w}^{a}\left[\begin{array}{c}
\delta \theta \\
\delta \gamma \\
\delta \psi
\end{array}\right]
$$

Where $C_{w}^{a}$ is calculated as

$$
\begin{aligned}
& C_{w}^{a}=\left[\begin{array}{ccc}
\cos \gamma & 0 & -\sin \gamma \cos \theta \\
0 & 1 & \sin \theta \\
\sin \gamma & 0 & \cos \gamma \cos \theta
\end{array}\right] \\
& {\left[\begin{array}{c}
\delta \theta \\
\delta \gamma \\
\delta \psi
\end{array}\right]=-\left(C_{w}^{a}\right)^{-1}\left(C_{b}^{n}\right)^{T}\left[\begin{array}{l}
\phi_{E} \\
\phi_{N} \\
\phi_{U}
\end{array}\right]}
\end{aligned}
$$

The sensitive measurement matrix is designed for the attitude measurements as

$$
H_{1}=\left[-\left(C_{w}^{a}\right)^{-1}\left(C_{b}^{n}\right)^{-1} \quad 0_{3 \times 6} \quad 0_{3 \times 6} \quad 0_{3 \times 3}\right]
$$


We consider the lever arm effect to improve the position and velocity accuracy of the integrated system. If $l^{b}$ is a lever arm vector from INS calibration centre to the GNSS antenna centre expressed in body frame, then GNSS position and velocity vectors can be expressed as.

$$
\begin{aligned}
& r_{g p s}^{n}=p+M_{r v} C_{b}^{n} l^{b} \\
& v_{g p s}^{n}=v_{i n s}^{n}+C_{b}^{n}\left(\omega_{e b}^{b} \times\right) l^{b}
\end{aligned}
$$

The measurement model for velocity and position is constructed as.

$$
\left.\begin{array}{l}
z_{1}=\tilde{p}-r_{g p s}^{n}=p+\delta p-r_{g p s}^{n} \\
z_{2}=\tilde{v}_{i n s}^{n}-v_{g p s}^{n}=v_{i n s}^{n}+\delta v^{n}-v_{g p s}^{n}
\end{array}\right\}
$$

Substituting Eq-48 in 46 \& 47, we get

$$
\begin{aligned}
& z_{1}=\delta p-M_{r v} C_{b}^{n} l^{b} \\
& z_{2}=\delta v^{n}-C_{b}^{n}\left(\omega_{e b}^{b} \times\right) l^{b}
\end{aligned}
$$

The sensitive measurement matrix for position and velocity is:

$$
H_{2}=\left[\begin{array}{ccccc}
0_{3 \times 3} & I_{3 \times 3} & 0_{3 \times 3} & 0_{3 \times 6} & -C_{b}^{n}\left(\omega_{e b}^{b} \times\right) \\
0_{3 \times 3} & 0_{3 \times 3} & I_{3 \times 3} & 0_{3 \times 6} & -M_{r v} C_{b}^{n}
\end{array}\right]
$$

The final measurement model for attitude, velocity and position measurements is obtained by combing Eq-45 and 51 as

$$
H=\left[\begin{array}{ccccc}
-\left(C_{w}^{a}\right)^{-1}\left(C_{b}^{n}\right)^{T} & 0_{3 \times 3} & 0_{3 \times 3} & 0_{3 \times 3} & 0_{3 \times 3} \\
0_{3 \times 3} & I_{3 \times 3} & 0_{3 \times 3} & 0_{3 \times 6} & -C_{b}^{n}\left(\omega_{e b}^{b} \times\right) \\
0_{3 \times 3} & 0_{3 \times 3} & I_{3 \times 3} & 0_{3 \times 6} & -M_{r v} C_{b}^{n}
\end{array}\right]
$$

\section{SIMULATION OF THE INTEGRATED SYSTEM}

The attitude, velocity and position measurements of GPS system are obtained from simulation of trajectory data using Matlab environment. A lower precision MEMS based IMU (STIM300) parameters are used to analyse the performance and validity of the integrated system through simulation results. The initial misalignment angles, with large heading angle are assumed to show the effectiveness of the GNSS attitude measurements. Simulation results are produced with; Gyro drift: 4 deg/hour, Gyro random walk: 1.7 $\mathrm{deg} / \sqrt{ }$ hour, Accelerometer bias: $1.5 \times 10^{-3} \mathrm{~g}$, Accelerometer random walk: $0.2 \times 10^{-3} \mathrm{~g}$, Lever arm vector: $\left[\begin{array}{ll}1 & 2\end{array}\right.$ $3]^{\mathrm{T}} \mathrm{m}$, GNSS attitude, velocity and position accuracy respectively are: $0.3 \mathrm{deg},\left[\begin{array}{lll}1 & 1 & 5\end{array}\right]^{\mathrm{T}} \mathrm{m}, 0.5 \mathrm{~cm} / \mathrm{sec}$.

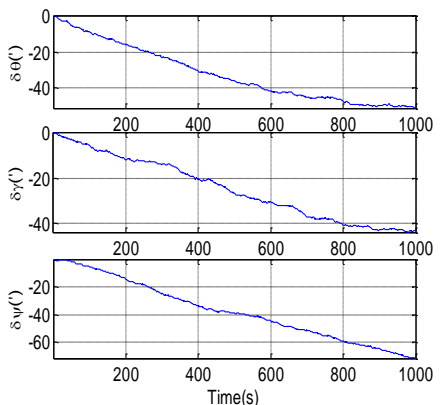

Figure 5. INS Attitude Error

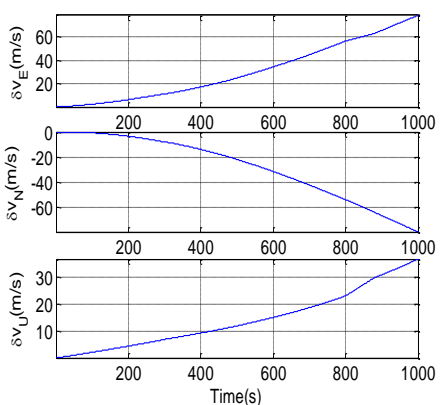

Figure 6. INS Velocity Error 


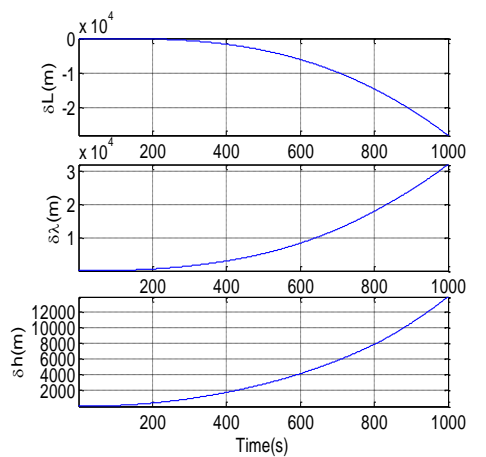

Figure 7 INS position error
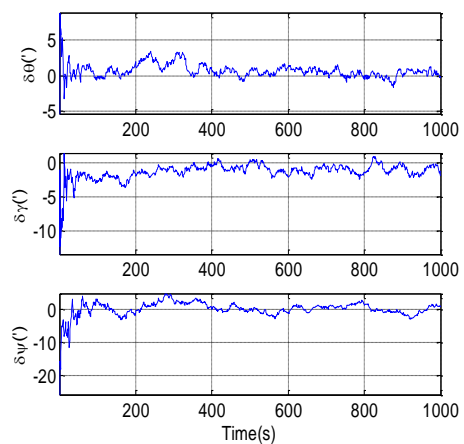

Figure 9 INS/GNSS attitude error

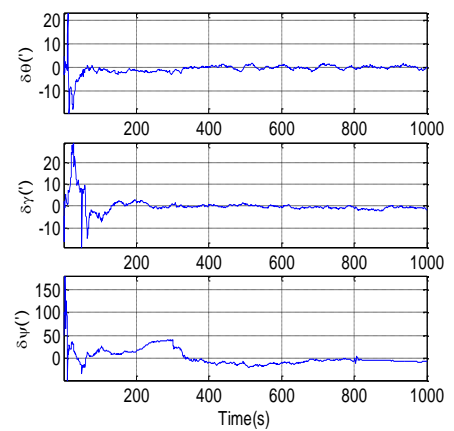

Figure 8 INS/GNSS attitude error
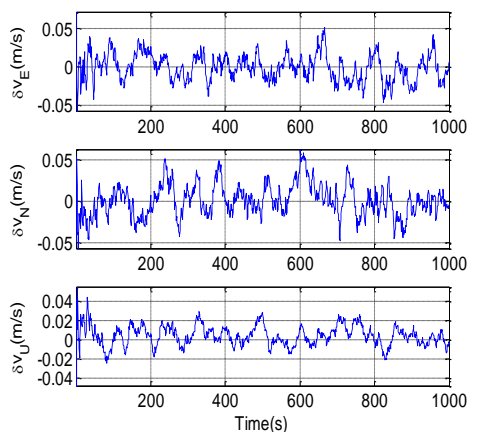

Figure 10 INS/GNS velocity error
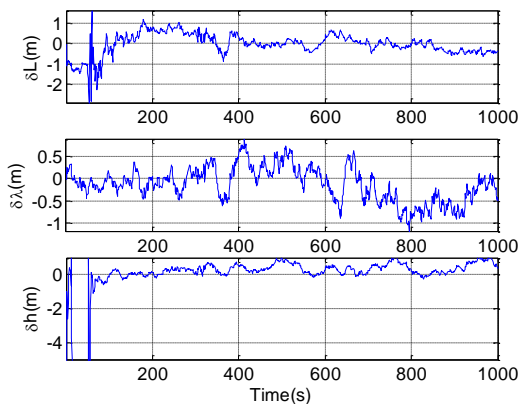

Figure 11 INS/GNSS position error

Figure 5, Figure 6 and Figure 7 show the standalone MEMS INS errors growth while Figure 8 shows the attitude error of the integrated system without using the attitude observation. Figure 9 illustrates the attitude error of the integrated system using the attitude measurement to the filter in the initial stage which showed a faster decrease in the attitude errors. The heading angle accuracy is improved which is very useful for the navigation system during nonaccelerating periods. Similarly the velocity and position errors of the integrated system shown in Figure 10 and Figure 11 are bounded to the cm level accuracy.

\section{CONCLUSION}

GNSS attitude, velocity and position from carrier phase measurements are mathematically derived and discussed for accurate integration. Loosely coupled approach is used to fuse the data of INS and GNSS using centralised LKF. A low accuracy MEMS based IMU (STIM300) parameters are used to generate the INS and GNSS data for simulation. Large attitude error is used to show the effectiveness of the GNSS attitude measurements. Simulation results showed that attitude errors decrease much faster than the filter without attitude measurements. The accuracy of heading angle is highly improved which is very important for initialization and for navigation systems during the benign or nonaccelerating period. There is a 
remarkable improvement in the navigation parameters due the precise velocity and position measurements from carrier phase measurements since velocity has direct coupling with the attitude error. After analysing the overall performance, INS/GNSS integrated system delivers more accurate results than can be achieved with the code phase or Doppler measurements. However, the carrier phase measurements for velocity and position estimation have the limitation of four satellites to be visible. Therefore, in urban or low visibility area the pseudo range and Doppler measurements are more effective and provide a continuous measurement to the filter for error estimation.

\section{REFERENCES}

[1] S. Godha, M. E. Cannon, GPS/MEMS INS Integrated System for Navigation in Urban Areas, GPS Solut 2007 11:193-203 DOI 10.1007/s10291-006-0050-8

[2] Poh, E., Koh, A. and Yu, X., Integration of Dead Reckoning Sensors with MEMS IMU. Proceeding of ION GPS 2002, pp.1148-1152

[3] Shin E., Estimation Techniques for Low-Cost Inertial Navigation. PhD Thesis, Department of Geomatics Engineering, University of Calgary, Canada, UCGE Report No. 20219, 2005

[4] Paul D. grove, Principle of GNSS, Inertial, and Multisensor Integrated Navigation Systems, ISBN-13:978-1-58053255-6, 2008

[5] Tazartes, D., et al., Synergistic Interferometric GPS-INS, Proc. ION NTM, Anaheim, CA, January 1995, pp. 657671

[6] Wagner, J. F., and G. Kasties, Modelling the Vehicle Kinematics as Key Element for the Design of Integrated Navigation System, Proc. IAIN World Congress, Berlin, Germany, October 2003

[7] Pierluigi Freda ,A. Angrisano ,S. Gaglione, S. Troisi, Time-differenced Carrier Phases Technique for Precise,GNSS Velocity Estimation, PS Solut 2015, 19:335-341 DOI 10.1007/s10291-014-0425-1

[8] Kaplan ED, Hegarty J., Understanding GPS: Principles and Applications, 2nd edn, 2006, Artech House, Norwood

[9] D.H. Titteron, J. Weston, Strapdown Inertial Navigation Technology, $2^{\text {nd }}$ Ed, Institute of Electrical Engineers, UK, 2005

[10] Kenneth R. Britting, Inertial Navigation Systems Analysis, Wiley-Interscience, New York, 1971

[11] Y. Qin, Inertial Navigation, Science Press, Beijing, China, 2009

[12] Aboelmagd Noureldin, Tashfeen B. Karamat, Jacques Georgy, Fundamentals of Inertial Navigation, Satellitebased Positioning and their Integration, Springer-Verlag Berlin Heidelberg 2013 